\title{
In vitro Elution of Penicillin, Ampicillin, Tetracycline, Tulathromycin, and Florfenicol From Plaster of Paris Beads
}

\author{
Paul Merkatoris ${ }^{1 *}$, Jennifer Schleining ${ }^{2}$, Adam Krull ${ }^{1}$, David Borts ${ }^{1}$ and Virginia Fajt ${ }^{3}$ \\ ${ }^{1}$ Department of Veterinary Diagnostic and Production Animal Medicine, College of Veterinary Medicine, lowa State University, \\ Ames, IA, United States, ${ }^{2}$ Department of Large Animal Clinical Sciences, College of Veterinary Medicine \& Biomedical \\ Sciences, Texas A\&M University, College Station, TX, United States, ${ }^{3}$ Department of Veterinary Physiology and \\ Pharmacology, College of Veterinary Medicine \& Biomedical Sciences, Texas A\&M University, College Station, TX, \\ United States
}

\section{OPEN ACCESS}

Edited by:

Yvonne A. Elce,

University of Prince Edward Island, Canada

Reviewed by:

Pierre-Yves Mulon,

The University of Tennessee, Knoxville,

United States

Jarred Mathew Williams,

University of Georgia, United States

*Correspondence:

Paul Merkatoris

pmerk@iastate.edu

Specialty section:

This article was submitted to

Veterinary Surgery and

Anesthesiology,

a section of the journal

Frontiers in Veterinary Science

Received: 20 July 2020 Accepted: 04 November 2020 Published: 30 November 2020

Citation:

Merkatoris P, Schleining J, Krull A, Borts D and Fajt $V$ (2020) In vitro

Elution of Penicillin, Ampicillin, Tetracycline, Tulathromycin, and Florfenicol From Plaster of Paris Beads. Front. Vet. Sci. 7:585423. doi: 10.3389/fvets.2020.585423
The objectives of this study were to report a recipe for making antibiotic impregnated Plaster of Paris (Al-PoP) beads using penicillin, ampicillin, tetracycline, tulathromycin, and florfenicol and to determine the in vitro elution rates of those antibiotics in the beads. The Al-PoP beads were made using Plaster of Paris powder, antibiotic, and water, cured for $24 \mathrm{~h}$, sterilized by ethylene oxide, and stored up to 5 months before testing. For each antibiotic, 20 beads were combined with bovine serum in sterile tubes and incubated at $37^{\circ} \mathrm{C}$ on a rocker. Serum was replaced at intervals over the 14 days study period, and antibiotic concentrations were determined by high pressure liquid chromatography with mass spectrometry. Separately, in a proof-of-concept study, the growth of $E$. coli and $T$. pyogenes in eluent from 10 beads for each antibiotic was quantified by flow cytometry. Antibiotic was detected in Al-PoP bead eluent for 14 days for all but the ampicillin beads, for which antibiotic was detected for 8 days. The concentration of antibiotic in eluent was greater than the minimum inhibitory concentration $(\mathrm{MIC})$ of tested bacteria for the entire study period for penicillin, tetracycline, tulathromycin, and florfenicol. The concentration of ampicillin remained greater than the MIC of E. coli for 4 days and T. pyogenes for 6 days. The colony forming units $(\mathrm{CFU}) / \mathrm{ml}$ of live $E$. coli and $T$. pyogenes was reduced over a 72-h period by 1-3 $\log _{10} \mathrm{CFU}$, with the exception of tetracycline, which reduced $\mathrm{CFU} / \mathrm{ml}$ of $T$. pyogenes by $<\log _{10} \mathrm{CFU}$. Al-PoP beads containing penicillin, tetracycline, tulathromycin, and florfenicol elute antibiotic well-above the MIC of selected isolates for the 2 weeks study period. These antibiotics show promise for delivery in joint and wound infections.

Keywords: Plaster of Paris, antibiotics, wound infection, elution, cattle

\section{INTRODUCTION}

Synovial sepsis, post-operative surgical site infections, and complicated wounds are a devastating cause of increased morbidity and mortality in large animal veterinary patients and can be career or even life-ending (1-3). While systemic antibiotics are the mainstay of treatment, complementary local antimicrobial therapies are often employed. 
These adjunct therapies are especially useful in the treatment of osteomyelitis, synovial sepsis, and surgical site infections (46). The benefits of local therapy include the ability to achieve local drug concentrations many times above bacterial minimum inhibitory concentration (MIC), avoidance of high systemic doses of antibiotics, and cost effectiveness (7-9).

The options for local antimicrobial therapy include intravenous and intraosseous regional limb perfusion $(10,11)$ and sustained release of antimicrobials eluted from implanted carriers such as collagen sponges (12), polymethylmethacrylate $(13,14)$, plaster of Paris (PoP) (7-9), constant rate infusion systems (15), and hydroxyapatite cement (13). Regional perfusions can achieve antimicrobial concentrations many times higher than the MIC of common pathogens, however use is limited to the distal extremity due to the need to place a tourniquet proximal to the area of interest and because high concentrations of drug are generally not maintained for very long after tourniquet removal. Antibiotics reported for use in regional perfusions in cattle include florfenicol, ampicillin-sulbactam, marbofloxacin, and ceftiofur (16-19). Constant rate infusion systems are also able to achieve high antimicrobial concentrations, however breakage or blockage of the intrasynovial catheter is common (15). Non-biodegradable polymethylmethacrylate implants are easy to use, however removal may be necessary. In addition, creating the implant produces an exothermic reaction, thus only heat stable antibiotics may be used (15). Biodegradable implants, including collagen sponges, hydroxyapatite cement, and PoP, offer the advantage of not requiring removal and being potentially more biocompatible. Purified type I collagen sponges are biocompatible, biodegradable, and are characterized by low immunogenicity (12). Collagen sponges and hydroxyapatite cement have the disadvantage of being more expensive than PoP. Plaster of Paris, or calcium sulfate hemihydrate, is inexpensive, readily available, and easy to use for local antimicrobial delivery (15). In addition, it is biocompatible, biodegradable, and osteoconductive when used in fracture repair and established osteomyelitis, providing a scaffold on which new bone formation can occur $(7,15)$. The in vitro elution of antibiotic from PoP beads has been reported for gentamicin, amikacin, clindamycin, enrofloxacin, vancomycin, tobramycin, and cefazolin (7$9,20,21)$. These studies show that the elution profile is different among antibiotics.

The use of antibiotic impregnated PoP (AI-PoP) beads constitutes an extra-label drug use under the Animal Medicinal Drug Use Clarification Act of 1994, which also prohibits or limits the use of previously studied drugs in food producing species. Antibiotics elected for use in this study include penicillin, ampicillin, tetracycline, tulathromycin, and florfenicol. These antibiotics were chosen because they are commonly used in food animal practice and extra-label use may be allowed.

The objectives of this study were to (1) report a recipe for making AI-PoP beads using penicillin, ampicillin, tetracycline,

\footnotetext{
Abbreviations: AI-PoP, Antibiotic impregnated Plaster of Paris; MIC, Minimum inhibitory concentration; CFU, Colony forming units; PoP, Plaster of Paris; MH, Mueller Hinton.
}

TABLE 1 | Antibiotic impregnated Plater of Paris bead recipes.

\begin{tabular}{|c|c|c|c|c|c|}
\hline & Penicillin & Tetracycline & Ampicillin & Tulathromycin & Florfenicol \\
\hline PoP powder (g) & 20 & 20 & 20 & 20 & 20 \\
\hline Antibiotic & $12 \mathrm{ml}$ & $2,000 \mathrm{mg}$ & $2,000 \mathrm{mg}$ & $3 \mathrm{ml}$ & $3 \mathrm{ml}$ \\
\hline Water (ml) & 0 & 8 & 9 & 5 & 6 \\
\hline Total antibiotic & 3.6 MIU & $2,000 \mathrm{mg}$ & $2,000 \mathrm{mg}$ & $300 \mathrm{mg}$ & $900 \mathrm{mg}$ \\
\hline Yield (beads) & 132 & 134 & 121 & 108 & 116 \\
\hline $\begin{array}{l}\text { Bead } \\
\text { concentration (per } \\
\text { bead) }\end{array}$ & $27 \mathrm{KIU}$ & $14.9 \mathrm{mg}$ & $16.5 \mathrm{mg}$ & $2.78 \mathrm{mg}$ & $7.76 \mathrm{mg}$ \\
\hline $\begin{array}{l}\text { Total antibiotic } \\
\text { tested (mg) }\end{array}$ & $288 \mathrm{MIU}$ & $1,192 \mathrm{mg}$ & $1,320 \mathrm{mg}$ & $222.4 \mathrm{mg}$ & $620.8 \mathrm{mg}$ \\
\hline
\end{tabular}

tulathromycin, and florfenicol and (2) determine the in vitro elution properties of the AI-PoP beads over 14 days.

We hypothesized that (1) the elution of all antibiotics from the AI-PoP beads would have a rapid initial phase and complete elution within 2 weeks and (2) the initial concentrations of antibiotic in the eluent would exceed the MIC of bacterial isolates used.

\section{MATERIALS AND METHODS}

Based on methods previously described $(7,9)$, AI-PoP beads were made on an open benchtop as follows: 20 grams of non-sterile PoP powder (Plaster of Paris, DAP Products Inc., Baltimore, MD) were thoroughly mixed with antibiotic powder or liquid in a specimen cup. When needed, water was added to achieve a pourable mixture (see Table 1 for weights and volumes used). Ampicillin (Polyflex ${ }^{\circledR}$, Boehringer Ingelheim Vetmedica Inc., St. Joseph, MO) and tetracycline (TC Vet $324^{\mathrm{TM}}$, Vetone, Manufactured for: MWI, Boise, ID) powder were used, whereas penicillin G procaine (PenOne Pro ${ }^{\mathrm{TM}}$, Norbrook Laboratories United, Newry, Northern Ireland), tulathromycin (Draxxin ${ }^{\circledR}$, Zoetic Inc., Kalamazoo, MI), and florfenicol (Norfenicol ${ }^{\circledR}$, Norbrook Laboratories Limited, Newry, North Ireland) were in solution. All AI-PoP bead ingredients were chosen for being inexpensive and easily accessible to general practice veterinarians, as it was desired to create a bead recipe that could be replicated and used in a general practice setting. Bead recipes were determined by maximizing the amount of antibiotic used with the outcome of an appropriately hardened bead, as too much antibiotic resulted in a bead that did not harden appropriately. In this study, all PoP powder used was derived from the same carton, all water was obtained from the same source in the hospital, and all antibiotics were derived from the same bottle or vial. All bead types used experimentally were created in a single batch, thus eliminating the need to control for variability in lot numbers. Each mixture was poured into a silicone candy bead mold (Chicago Culinary FX, Westchester, IL) and a wooden tongue depressor was used to completely fill the wells. The use of a silicone bead mold allowed standardization of bead size in this study. The mold created uniform, $6 \mathrm{~mm}$ diameter beads, yielding a surface area of $113 \mathrm{~mm}^{2}$ and a volume of $113 \mathrm{~mm}^{3}$. The AI-PoP beads were allowed to cure in the mold for $24 \mathrm{~h}$ at 
room temperature (with the exception of penicillin beads, which were cured at $4^{\circ} \mathrm{C}$ ), sterilized by ethylene oxide, and stored at room temperature (with the exception of penicillin beads, which were stored at $4^{\circ} \mathrm{C}$ based on storage conditions identified on the drug label). Testing was completed within 5 months of AI-PoP bead manufacturing.

For each antibiotic, 20 AI-PoP beads and seven milliliters of bovine serum (Lampire Biological Laboratories, Inc., Pipersville, PA) were aseptically transferred to individual sterile polypropylene test tubes. The eluent from each AI-PoP bead type was tested in quadruplicate; four test tubes of AI-PoP beads were tested for each antibiotic. The number of beads, choice of serum, and volume of serum were chosen based on previous studies $(7,9)$ and because it was sufficient to immerse the beads in serum. The minimal volume necessary to submerge the 20 beads in the $50 \mathrm{~mL}$ polypropylene test tubes was $5 \mathrm{~mL}$. Two additional $\mathrm{mL}$ of bovine serum were added during the first 4 days of the experiment to mimic the increase of effusion occurring in the early phase of the infection. The tubes were maintained in a rotating incubator at $37^{\circ} \mathrm{C}$, except when removing eluent and adding serum. The entire volume of eluent was removed at specified time points and replaced with fresh bovine serum. A volume of $7 \mathrm{~mL}$ was initially used at $4,8,12,24,36,48$, and $72 \mathrm{~h}$. This volume was subsequently reduced to $5 \mathrm{~mL}$ at $96,120,144$, 192,264 , and $336 \mathrm{~h}$ in order to mimic the reduction of effusion that clinically occurs during the healing period. Eluent samples were stored at $-70^{\circ} \mathrm{C}$ until analyzed ( $\sim 1$ month).

Concentrations of five antibiotics were determined using high-pressure liquid chromatography with mass spectrometry detection after protein precipitation of samples with acetonitrile. High-pressure liquid chromatography with mass spectrometry detection was performed using an Agilent 1100 Pump, column compartment, and autosampler (Santa Clara, CA, USA) coupled to an ion trap mass spectrometer (LTQ, Thermo Scientific, San Jose, CA, USA). Serum samples, serum spikes, serum quality controls, and bovine serum blanks, 50 or $100 \mu \mathrm{L}$, depending on calibration curve concentrations of the antibiotic, were mixed with $450 \mu \mathrm{L}$ of acetonitrile to precipitate serum proteins. The acetonitrile contained an internal standard at a concentration of either 250 or $1,000 \mathrm{ng} / \mathrm{mL}$, depending on calibration curve concentrations of the antibiotic. The samples were vortexed for $5 \mathrm{~s}$ and centrifuged for $10 \mathrm{~min}$ at 7,500 rpm to sediment the protein pellet. A portion, 20 or $50 \mu \mathrm{L}$, of the supernatant was then diluted with water to $1 \mathrm{~mL}$ of volume in an autosampler vial. Further dilution of the samples, if needed, was done with blank serum that had been precipitated and diluted identically as the samples. These dilutions were done in autosampler vials fitted with glass inserts and dilutions were 1:5 or 1:10. The vials were then centrifuged at 2,400 rpm prior to analysis. All the quality control samples passed the tolerance of being within $\pm 15 \%$ of the nominal concentration. The limit of quantitation of the analysis was $0.5 \mu \mathrm{g} / \mathrm{mL}$ with a limit of detection of $0.1 \mu \mathrm{g} / \mathrm{mL}$.

The mean observed peak of antibiotic elution was calculated for each antibiotic. The amount of antibiotic eluted was displayed in two different ways: first, as the amount of antibiotic eluted in the first $72 \mathrm{~h}$, expressed as a percent of antibiotic released over the 2-weeks study period, and second, as the total amount of antibiotic released over the 2-weeks study period, expressed as a percent of total incorporated antibiotic. The concentration of antibiotic within the eluent was compared to the MICs of the bacterial isolates used in this study. Some authors consider time dependent antibiotics, such as those used in this study, to be most efficacious when concentration is maintained above the MIC for at least $50 \%$ of the dosing interval (22), thus, we chose to compare the antibiotic concentration to the MIC of the selected isolates. The elution half-life was calculated based on the loglinear regression of the terminal portion of the concentrationtime curve using standard pharmacokinetic software (Certera Phoenix 64 8.1.0.3530). The elution half-life is the interval of time required for the concentration of antibiotic to be decreased by $50 \%$.

The quantification of bacteria subjected to AI-PoP eluent was evaluated against two bacterial isolates, an American Type Culture Collection strain of E. coli and a clinical isolate of $T$. pyogenes. These are two of the most common isolates in cattle from cases of synovial sepsis (23). Together they encompass a broad spectrum of bacteria, $T$. pyogenes being a slow growing Gram-positive facultative anaerobe, and E. coli being a fastidious Gram-negative facultative anaerobe $(24,25)$. In this proofof-concept phase of the study, a single test of each AI-PoP and bacteria combination was completed. Using the McFarland standard, colonies of pure cultures of E. coli and T. pyogenes were used to inoculate test tubes containing ten AI-PoP beads and five milliliters of Mueller Hinton (MH) broth to a concentration of $10^{7} \mathrm{CFU} / \mathrm{mL}$. This concentration was elected because it was the most concentrated inoculum that could be reasonably achieved using these methods. It has been shown that the risk for surgical site infection is markedly increased when contaminated with more than $10^{5}$ microorganisms per gram of tissue (26). Thus, the inoculation dose of $10^{7}$ was considered sufficient to represent clinical infection. A single negative control test of $\mathrm{MH}$ broth lacking AI-PoP beads was also inoculated at the same concentration for each bacterium. Following inoculation, the tubes were maintained in a rotating incubator at $37^{\circ} \mathrm{C}$. Twenty-four, 48, and $72 \mathrm{~h}$ after inoculation, a one milliliter sample of eluent was obtained for quantification of live and dead bacteria by flow cytometry, and the volume was replaced with sterile $\mathrm{MH}$ broth. Live and dead bacteria in samples were stained for quantification by flow cytometry using a commercially available kit (Molecular Probes, Inc.; Eugene, OR). Samples were prepared according to manufacturer's instruction, then immediately transported to the flow cytometry laboratory for quantification. The number of events in the bead region was set to 5,000 and the sample was assayed at $488 \mathrm{~nm}$. The data were processed by framing the region around the live and dead populations to yield events data for live and dead bacteria. The bacterial culture density was calculated using outcome events data and dilution factors.

\section{RESULTS}

All AI-PoP beads eluted detectable concentrations of antibiotic for the 14-days sampling period, with the exception of ampicillin, 

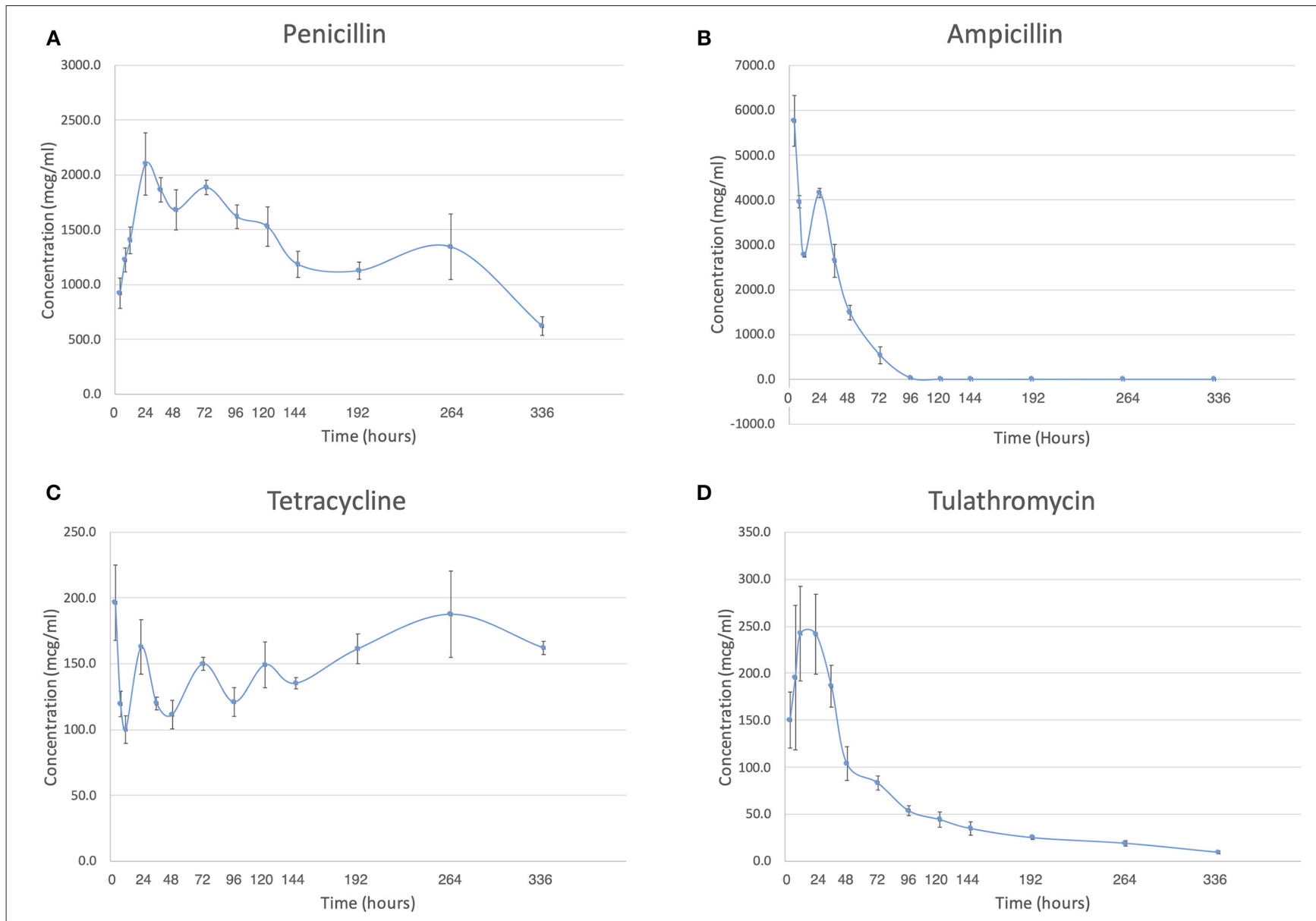

E

Florfenicol

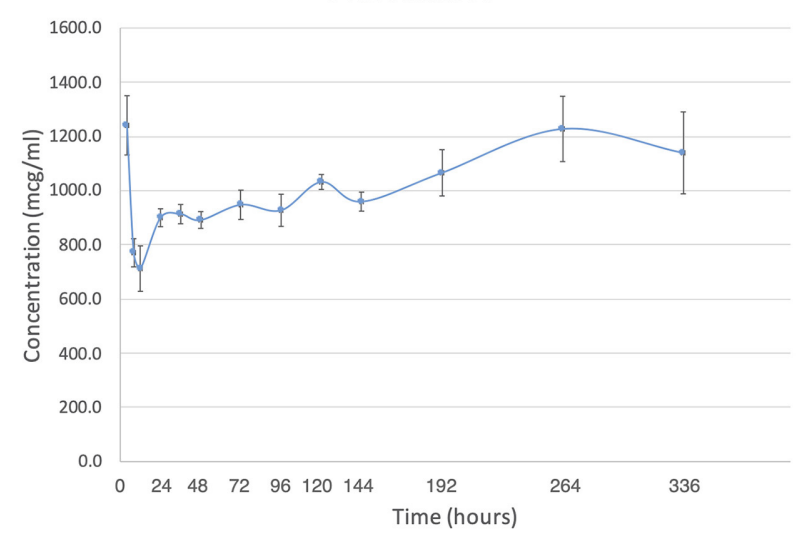

FIGURE 1 | The concentration in $\mathrm{mcg} / \mathrm{ml}$ is shown over the two-week study period for each antibiotic. (A) Penicillin. (B) Ampicillin. (C) Tetracycline. (D) Tulathromycin. (E) Florfenicol.

which eluted detectable concentrations of antibiotic for 8 days. Mean observed peak ampicillin, florfenicol, and tetracycline concentrations occurred at $4 \mathrm{~h}$, mean observed peak tulathromycin concentration occurred at $12 \mathrm{~h}$, and mean observed peak penicillin concentration occurred at $24 \mathrm{~h}$ (Figure 1).

The amount of antibiotic released in the first $72 \mathrm{~h}$, expressed as a percent of antibiotic released over the 2-weeks study period, 
TABLE 2 | Mean percent elution in terms of total included antibiotic and antibiotic eluted over the 14 day study period.

\begin{tabular}{lcccc}
\hline & $\mathbf{1 4} \mathbf{d} /$ total & Std dev & $\mathbf{7 2 ~ h / 1 4 ~ d ~}$ & Std dev \\
\hline Ampicillin & 45.2 & 2.1 & 99.9 & 0.04 \\
Florfenicol & 49.2 & 2.2 & 58.4 & 0.8 \\
Penicillin & 33.6 & 0.9 & 67.6 & 2.1 \\
Tetracycline & 3.8 & 0.1 & 59.4 & 1.4 \\
Tulathromycin & 16.8 & 1.8 & 89.9 & 1.5
\end{tabular}

TABLE 3 | Minimum inhibitory concentration (micrograms $/ \mathrm{mL}$ ) of isolates used, provided by the lowa State University Veterinary Diagnostic Lab.

\begin{tabular}{lcc}
\hline & E. coli & T. pyogenes \\
\hline Ampicillin & 4.00 & 0.25 \\
Florfenicol & 4.00 & 1.00 \\
Tetracycline & 1.00 & 4.00 \\
Penicillin & $>8.00$ & $<=0.12$ \\
Tulathromycin & 8.00 & $<=1.00$ \\
\hline
\end{tabular}

TABLE 4 | Elution half-life (in hours) of antibiotics in Al-PoP beads incubated for 14 days in bovine serum.

\begin{tabular}{lccccc}
\hline & Rep 1 & Rep 2 & Rep 3 & Rep 4 & Mean \\
\hline Ampicillin & 8.8 & 9.4 & 12.0 & 8.5 & 9.6 \\
Penicillin & 248 & 236 & 185 & 216 & 221.2 \\
Tulathromycin & 101 & 101 & 124 & 95 & 105.4 \\
Florfenicol & Could not be calculated because slope was not negative \\
Tetracycline & Could not be calculated because slope was not negative \\
\hline
\end{tabular}

was greatest for ampicillin and least for florfenicol (Table 1). The total amount of antibiotic released over the 2-weeks study period, expressed as a percent of total incorporated antibiotic, was greatest for florfenicol and least for tetracycline (Table 2).

The MIC data for each antibiotic and bacterial strain, as provided by the Iowa State University Veterinary Diagnostic Laboratory, is shown in Table 3. The concentration of antibiotic within the eluent reported at each time point was greater than the MIC of both E. coli and T. pyogenes for penicillin, tetracycline, tulathromycin, and florfenicol for the entire study period. The concentration of ampicillin remained greater than the MIC of $E$. coli for 4 days and T. pyogenes for 6 days.

Ampicillin had the shortest elution half-life, and penicillin had the longest (Table 4). The elution half-life of florfenicol and tulathromycin could not be calculated in any of the samples because the slope was not negative.

The results of the flow cytometry proof-of-concept study are shown in Figures 2A,B. The decrease in concentrations observed at 24 and 48 h of growth represents the decrease in concentrations that resulted from adding $1 \mathrm{ml}$ of $\mathrm{MH}$ broth to the culture tube following sampling. The colony forming units (CFU)/ml of $E$. coli is reduced by $\sim 1-2 \log _{10} \mathrm{CFU}$ by $72 \mathrm{~h}$ for all antibiotics compared to the control sample. The CFU/ml of $T$. pyogenes is reduced by $3 \log _{10}$ CFU for all antibiotics except tetracycline by $72 \mathrm{~h}$ compared to the control sample. CFU reduction in the tetracycline sample was $<\log _{10} \mathrm{CFU}$.

\section{DISCUSSION}

This study was successful in creating antibiotic impregnated beads using five different antibiotics. The first hypothesis, that the elution of all antibiotics from the AI-PoP beads would have a rapid initial phase and complete elution within 2 weeks, was rejected. Only the elution of ampicillin was complete within 2 weeks; the remaining antibiotics eluted antibiotic for the full 2 -weeks study period. The second hypothesis, that the initial concentrations of antibiotic in the eluent would exceed the MIC of bacterial isolates used, was accepted. The concentration of antibiotic in the eluent exceeded the MIC of bacterial isolates for a minimum of 4 days.

The amount of antibiotic eluted by the AI-PoP beads was low in this study, ranging from 3.79 to $49.23 \%$. The amount of antibiotic eluted has been shown to be proportional to dissolution of the bead, with the rate of dissolution being affected by increases in porosity (27). Porosity, determined by scanning electron microscopy (28), and rate of bead dissolution were not analyzed in this study because the ideal porosity and other biomaterials properties for AI-PoP beads remains unknown. The authors hypothesize that factors such as water solubility of the antibiotic and molecular binding properties with the PoP may have played a role in the differences in amount of antibiotic eluted. It has been speculated that factors such as storage temperature and humidity, length of time stored, and combination of different antibiotics used may have an effect on bead dissolution. The length of time stored prior to elution in this study was 5 months, and was 6 months in a previous study (7). It remains unknown what the maximum length of time these products may be considered shelf-stable; however, the products used in this study remained effective following 5 months of storage.

The elution pattern of penicillin, ampicillin, and tulathromycin in our study is similar to the previously reported elution pattern of most antibiotics $(7,9)$, characterized by an initial rapid release of antibiotic, subsequently followed by low concentration release for the remainder of the study period. The elution pattern of tetracycline and florfenicol is atypical in that a relatively constant level of elution was maintained for the entire study period. This pattern has previously been reported for enrofloxacin and was attributed to the drug having a low solubility in water (9). This may explain the elution pattern of florfenicol, as it also has a low solubility in water. However, tetracycline is soluble in water, and the authors suspect that there is an unknown molecular interaction between the PoP and tetracycline that is responsible for the elution pattern that was observed. One hypothesis is that oxytetracycline is known to chelate calcium ions, thus is may be chelating calcium present in the Plaster of Paris. The authors hypothesize that the longer duration of time between sampling times later in the study led to relatively higher levels of antibiotic in the eluent for tetracycline and florfenicol. 

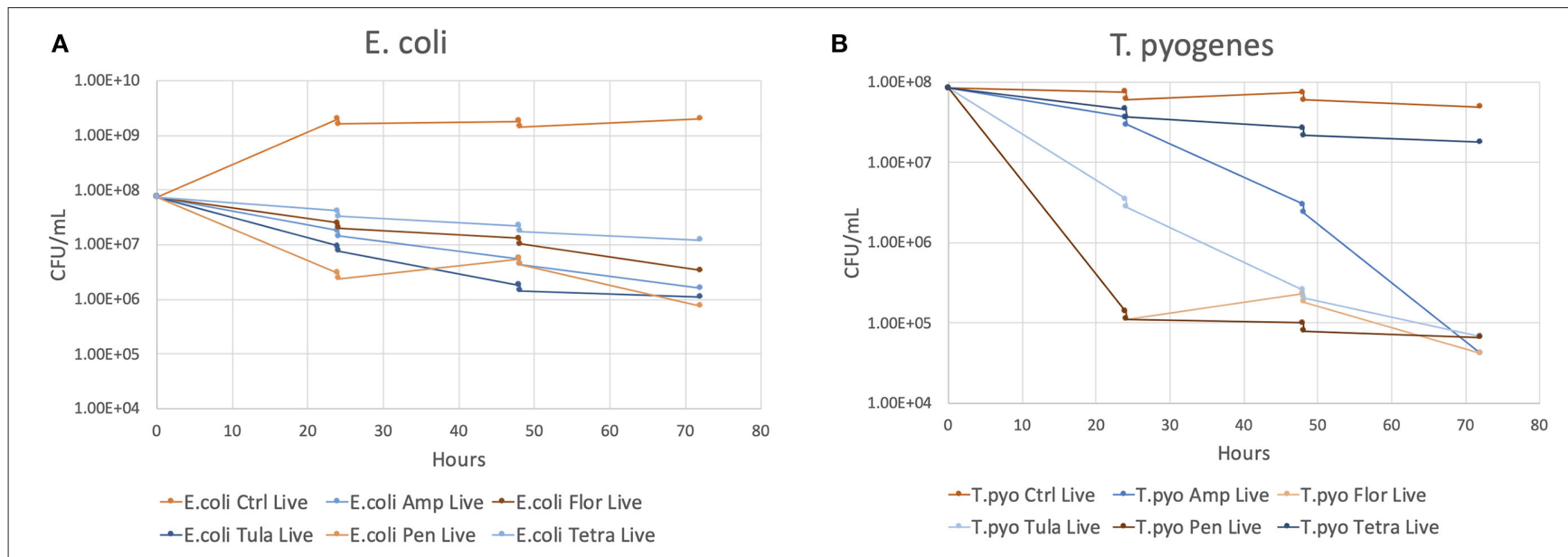

FIGURE 2 | The CFU/ml of each antibiotic and the control at 24, 48, and $72 \mathrm{~h}$ of growth. (A) E. coli. (B) T. pyogenes.

This study is consistent with previous reports that found a variable volume of liquid is needed for individual batches in order to obtain an appropriate antibiotic-PoP mixture consistency prior to filling the bead mold $(7,9)$. Because of individual properties associated with each antibiotic, each AI-PoP bead preparation was different. Because procaine penicillin $G$ was used, penicillin beads were cured and stored in a refrigerator in accordance with the handling instructions of the drug. It is unknown whether this was necessary, or if curing and storage at room temperature would have been acceptable.

A sampling period of 2 weeks was chosen in this study. Based on the rapid elution of antibiotic from AI-PoP beads observed in a previous study using PoP beads (7), a similar elution profile was anticipated. However, we were surprised that in the current study, AI-PoP beads eluted antibiotic for the entire 14-days study period, with the exception of ampicillin. In previous studies using PoP beads, antibiotics such as gentamicin, amikacin, and clindamycin exhibited a rapid elution profile, while vancomycin, enrofloxacin, and tobramycin exhibited a prolonged release elution profile $(7-9,16)$.

Previous studies have used eluent to qualitatively describe bacterial inhibition $(7,8)$. Flow cytometry has been shown to be an accurate and rapid method of determination of the concentration of bacteria in a sample while distinguishing between live and dead organisms (29). This proof-of-concept portion of the study serves to show that flow cytometry can be used to quantitatively describe the effect of AI-PoP eluent on bacterial inhibition.

The choice of antibiotics for systemic administration should be based upon culture and antimicrobial susceptibility data, but because of a variety of factors including cost, time to achieve culture and sensitivity results, negative growth on cultures, and other reasons, the choice is often based upon clinical experience, empirical data, and commonly known bacterial isolates associated with specific conditions. The choice for local delivery of antibiotics is based upon similar criteria, although MIC breakpoints used to identify susceptible and resistant isolates are not predictive when antibiotic drugs are applied topically or locally, since achievable concentrations and effects on clinical outcome are unknown. Additional factors that could potentially influence the performance of local antimicrobials include bacterial burden, wound contamination, and wound exudate. It remains unknown how this in vitro work is to relate in vivo. There are many factors of in vivo infection that were not replicated by this study, including microbe-related risk factors such as biofilm, and host-related factors such as an immune response.

The antibiotics chosen for this study were selected because they are labeled for use in food producing species and their extralabel use may be allowed. Food producing species are subject to restrictions on drug use such as provisions within the Animal Medicinal Drug Use Clarification Act of 1994. It is recommended that the Food Animal Residue Avoidance Database be consulted for any extralabel drug use, including those described in this report. It is unknown what the serum or tissue concentrations of these drugs might be after application of AI-PoP bead, so caution should be used when predicting withdrawal times.

A limitation of this study is that elution was only investigated for 2 weeks. Elution data showed that a longer period would have been beneficial for penicillin, tetracycline, tulathromycin, and florfenicol. Another limitation is that the mechanical properties of the PoP beads were not characterized, and we were not able to provide further information regarding the rate of dissolution of the beads. A final limitation of this study is the absence of multiple specimens in the proof-of-concept study to determine the in vitro inhibition of bacteria by AI-PoP eluent.

Future studies should focus on the in vivo application of penicillin, tetracycline, tulathromycin, and florfenicol AI-PoP beads to corroborate the results of this in vitro study. These antibiotics appear to perform well in eluting antibiotic from AI-PoP beads and show promise for use in food animal species. 


\section{DATA AVAILABILITY STATEMENT}

The raw data supporting the conclusions of this article will be made available by the authors, without undue reservation.

\section{AUTHOR CONTRIBUTIONS}

$\mathrm{PM}, \mathrm{JS}, \mathrm{AK}$, and $\mathrm{DB}$ contributed to the design and completion of this study. VF performed the elution half-life analysis. All authors contributed to the writing, editing, and final approval of the manuscript.

\section{REFERENCES}

1. Richardson DW, Ahern BJ. Synovial and Osseous Infections. In: Auer JA, Stick JA, editors. Equine Surgery, 4th Edn. St. Louis, MO: Saunders Elsevier (2012). p. 1195-200. doi: 10.1016/B978-1-4377-0867-7.00 085-5

2. McIlwraith CW. Treatment of infectious arthritis. Vet Clin North Am Large Anim Pract. (1983) 5:363-79. doi: 10.1016/S0196-9846(17)30083-6

3. Schneider RK, Bramlage LR, Moore RM, Mecklenburg LM, Kohn CW, Gabel AA. A retrospective study of 192 horses affected with septic arthritis/tenosynovitis. Equine Vet J. (1992) 24:436-42. doi: 10.1111/j.2042-3306.1992.tb02873.x

4. Rubio-Martìnez LM, Cruz AM. Antimicrobial regional limb perfusion in horses. J Am Vet Med Assoc. (2006) 228:706-12. doi: 10.2460/javma.228.5.706

5. Whitehair KJ, Adams SB, Parker JE, Blevins WE, Fessler JF. Regional limb perfusion with antibiotics in three horses. Vet Surg. (1992) 21:28692. doi: 10.1111/j.1532-950X.1992.tb00066.x

6. Whithair KJ, Bowersock TL, Blevins WE, Fessler JF, White MR, Van Sickle DC. Regional limb perfusion for antibiotic treatment of experimentally induced septic arthritis. Vet Surg. (1992) 21:367-73. doi: 10.1111/j.1532-950X.1992.tb01713.x

7. Santschi EM, McGarvey L. In vitro elution of gentamicin from plaster of Paris beads. Vet Surg. (2003) 32:128-33. doi: 10.1053/jvet.2003.50010

8. Atilla A, Boothe HW, Tollett M, Duran S, Diaz DC, Sofge J, et al. In vitro elution of amikacin and vancomycin from impregnated plaster of Paris beads. Vet Surg. (2010) 39:715-21. doi: 10.1111/j.1532-950X.2009.00632.x

9. Phillips H, Booth DM, Bennet RA. Elution of clindamycin and enrofloxacin from calcium sulfate hemihydrate beads In vitro. Vet Surg. (2015) 44:100311. doi: $10.1111 /$ vsu. 12415

10. Pille F, De Baere S, Ceelen L, Dewulf J, Croubles S, Gasthuys F, et al. Synovial fluid and plasma concentrations of ceftiofur after regional intravenous perfusion in the horse. Vet Surg. (2005) 34:610-7. doi: 10.1111/j.1532-950X.2005.00095.x

11. Mattson S, Bouré L, Pearce S, Hurtig M, Burger J, Black W. Intraosseous gentamicin perfusion of the distal metacarpus in standing horses. Vet Surg. (2004) 33:180-6. doi: 10.1111/j.1532-950x.2004.04026.x

12. Ivester KM, Adams SB, Moore GE, Van Sickle DC, Lescun TB. Gentamicin concentrations in synovial fluid obtained from the tarsocrural joints of horses after implantation of gentamicin-impregnated collagen sponges. Am J Vet Res. (2006) 67:1519-26. doi: 10.2460/ajvr.67.9.1519

13. Ethell MT, Bennett RA, Brown MP, Mettitt K, Davidson JS, Tran $\mathrm{T}$. In vitro elution of gentamicin, amikacin, and ceftiofur from polymethylmethacrylate and hydroxyapatite cement. Vet Surg. (2000) 29:375-82. doi: 10.1053/jvet.2000.7535

14. Phillips H, Boothe DM, Shofer F, Davidson JS, Bennett RA. In vitro elution studies of amikacin and cefazolin from polymethylmethacrylate. Vet Surg. (2007) 36:272-8. doi: 10.1111/j.1532-950X.2007.00262.x

15. Cruz AM, Rubio-Martinez L, Dowling T. New antimicrobials, systemic distribution, and local methods of antimicrobial delivery in horses. Vet Clin North Am Equine Pract. (2006) 22:297-322. doi: 10.1016/j.cveq.2006.03.006

\section{FUNDING}

This project was funded by the American College of Veterinary Surgery Foundation- Surgeon-in-training grant (2018).

\section{ACKNOWLEDGMENTS}

This manuscript has been released as a pre-print at BMC Veterinary Research (30).

16. Depenbrock SM, Simpson KM, Niehaus AJ, Lakritz J, Papich MG Pharmacokinetics of ampicillin-sulbactam in serum and synovial fluid samples following regional intravenous perfusion in the distal portion of a hind limb of adult cattle. Am J Vet Res. (2017) 78:13729. doi: 10.2460/ajvr.78.12.1372

17. Gilliam JN, Streeter RN, Papich MG, Washburn KE, Payton ME. Pharmacokinetics of florfenicol in serum and synovial fluid after regional intravenous perfusion in the distal portion of the hind limb of adult cows. Am J Vet Res. (2008) 69:997-1004. doi: 10.2460/ajvr.69.8.997

18. Celani G, Tulini SMR, Montesano C, Zezza D, Sergi M, Varasano V, et al. Pharmacokinetics of marbofloxacin administered via intravenous regional limb perfusion in dairy cows: evaluation of two different tourniquets. Vet Rec Open. (2017) 4:e000227. doi: 10.1136/vetreco-2017-000227

19. Navarre CB, Zhang L, Sunkara G, Duran SH, Kompella UB. Ceftiofur distribution in plasma and joint fluid following regional limb injection in cattle. J Vet Pharmacol Therap. (1999) 22:13-9. doi: 10.1046/j.1365-2885.1999.00186.x

20. Sanicoli SM, Albert SF. The In vitro elution characteristics of vancomycin and tobramycin from calcium sulfate beads. J Foot and Ankle Surg. (2005) 44:121-4. doi: 10.1053/j.jfas.2005.01.006

21. Udomkusonsri P, Kaewmokul S, Arthitvong S, Phaochoosak N. Elution profiles of cefazolin from PMMA and calcium sulfate beads prepared from commercial cefazolin formulations. J Vet Med Sci. (2012) 74:3015. doi: 10.1292/jvms.11-0095

22. Papich MG. Pharmacokinetic-pharmacodynamic (PK-PD) modeling and the rational selection of dosage regimes for the prudent use of antimicrobial drugs. Vet Microbiol. (2014) 171:480-6. doi: 10.1016/j.vetmic.2013.12.021

23. Desrochers A, Francoz D. Clinical management of septic arthritis in cattle. Vet Clin Food Anim. (2014) 30:177-203. doi: 10.1016/j.cvfa.2013.11.006

24. Biberstein EL, Hirsch DC. Corynebacteria; Arcanobacterium (Actinomyces) pyogenes; Rhodococcus equi. In: Hirsch DC, Zee YC, editors. Veterinary Microbiology. Malden, MA: Blackwell Science, Inc (1999). p. 127-34.

25. Hirsch DC. Escherichia. In: Hirsch DC, Zee YC, editors. Veterinary Microbiology. Malden, MA: Blackwell Science, Inc (1999). p. 69-74.

26. Stewart S, Richardson DW. Surgical site infection and the use of antimicrobials. In Auer JA, Stick JA, editors. Equine Surgery, 4th Edn. St. Louis, MO: Saunders Elsevier (2012). p. 68-84.

27. Roberts R, McConoughey SJ, Calhoun JH. Size and composition of synthetic calcium sulfate beads influence dissolution and elution rates In vitro. J Biomed Mater Res B. (2014) 102B:667-73. doi: 10.1002/jbm.b.33045

28. Seddighi MR, Griffon DJ, Constable PD, Martin-Jimenez T. Effects of porcine small intestinal submucosa on elution characteristics of gentamicin-impregnated plaster of Paris. Am J Vet Res. (2007) 68:1717. doi: 10.2460/ajvr.68.2.171

29. Orth R, O’Brien-Simpson N, Dashper S, Walsh K, Reynolds E. An efficient method for enumerating oral spirochetes using flow cytometry. J Microbiol Methods. (2010) 80:123-8. doi: 10.1016/j.mimet.2009.11.006

30. Merkatoris $\mathrm{P}$, Schleining J, Krull A, Borts D, Fajt V. In vitro elution and E. coli and T. pyogenes killing profile of penicillin, 
ampicillin, tetracycline, tulathromycin, and florfenicol from Plaster of Paris beads. Res Square. [preprint]. (2020). doi: 10.21203/rs.3.rs-24 $056 / \mathrm{v} 1$

Conflict of Interest: The authors declare that the research was conducted in the absence of any commercial or financial relationships that could be construed as a potential conflict of interest.
Copyright $\odot 2020$ Merkatoris, Schleining, Krull, Borts and Fajt. This is an openaccess article distributed under the terms of the Creative Commons Attribution License (CC BY). The use, distribution or reproduction in other forums is permitted, provided the original author $(s)$ and the copyright owner(s) are credited and that the original publication in this journal is cited, in accordance with accepted academic practice. No use, distribution or reproduction is permitted which does not comply with these terms. 